\title{
Control strategies for friction dampers: numerical assessment and experimental investigations.
}

\author{
H.T. Coelho ${ }^{1}$, M.B. Santos ${ }^{1}$, F.P. Lepore Neto ${ }^{1}$ and J. Mahfoud ${ }^{2}$. \\ ${ }^{1}$ Mechanical System Laboratory, Mechanical Engineering School, Federal University of Uberlandia, Brazil. \\ ${ }^{2}$ LaMCoS, Insa Lyon, 113227A - Building J. d'Alembert - 2ème, France
}

\begin{abstract}
The use of friction dampers has been proposed in a wide variety of mechanical systems for which it is not possible to apply viscoelastic materials, fluid based dampers or others viscous dampers. An important example is the application of friction dampers in aircraft engines to reduce the blades vibration amplitudes. In most cases, friction dampers have been studied in a passive way, however, a significant improvement can be achieved by controlling the normal force in the dampers. The aim of this paper is to study three control strategies for friction dampers based on the hysteresis cycle. The first control strategy maximizes the energy removal in each harmonic oscillation cycle, by calculating the optimum normal force based on the last displacement peak. The second control strategy combines the first one with the maximum energy removal strategy used in the smart spring devices. Finally, is presented the strategy which homogenously modulates the friction force. Numerical studies were performed with these three strategies defining the performance metrics. The best control strategy was applied experimentally. The experimental test rig was fully identified and its parameters were used for the numerical simulations. The obtained results show the good performance for the friction damper and the selected strategy.
\end{abstract}

\section{Introduction}

The use of friction dampers has been proposed in a wide variety of mechanical systems for which it is not possible to apply viscoelastic materials, fluid based dampers or others viscous dampers. A first example is the application of friction dampers in aircraft engines to reduce the blades vibration amplitudes [1]. Seismic isolation based on friction was proven be a very useful solution for large civil structures [2]. In most cases, friction dampers had been studied in a passive way, however, a significant improvement can be achieved by controlling the normal force in the dampers [3]. Friction dampers with variable normal force are classified as semi-active devices and their appeal is to have performance levels rivalling the active devices with low level of energy consumption [4]. A brief bibliography review should reveal numerous strategies for control laws in friction damping. Inaudi [5] proposed that the normal force should be proportional to the absolute value of the prior local peak of damper deformation, the author called damper deformation the relative displacement of the degrees of freedom, which between them the damper was installed. The best proportion for the normal force was described by Menq $[6,7]$. Ozbulut et al [8] proposed the use of fuzzy logic to actualize the normal force in semi active friction dampers used for huge civil structures. Yang et al [9] designed a modification for the Inaudi [5] control strategy. This modification actualizes the normal force continuously with the damper deformation. The authors proposed two parameters to adjust their control strategy: Control gain and a boundary layer parameter which means how fast the controller changes the normal force. The aforementioned control strategy demand high levels of control gain, as shown the results obtained by the authors. Nitzsche et al [10] propose a control strategy for system where the friction damper has two states only: "ON" and "OFF". In their strategy, the damper accumulates potential energy, by means of the tangential elastic deformation. Therefore, the damper accumulates energy only when the system goes far from its static equilibrium position, this energy is not restored to the system since the damper is turned off when it moves towards its static 
equilibrium position. The authors conclude that a passive friction damper can be optimized only for specific excitation forces and system dynamics characteristics. Besides, Vanderborght et al [11] define the challenge as tune the friction dampers to achieve any desired damping characteristics, pointing out the main advantage as the high damping forces even for low velocities.

The aim of this paper is to study four cases of control strategies for friction dampers based on the hysteresis cycle. The first control strategy, named "Case A", maximizes the energy removal in each harmonic oscillation cycle calculating the optimum normal force, i.e., uses the force that maximizes the hysteresis area reducing the vibration amplitude. A second control law, named "Case B", combines the first strategy with the maximum energy removal strategy used in the smart spring devices. Control law namely "Case C" has been proposed by Yang et al [9]. Finally, is presented a modification in the modulated homogeneous friction damper as it has been originally proposed, namely "Case D". Case "D" maintains the hysteresis cycle for case C, changes had been done introducing the future speed predictor. This modification intends to reduce the necessity for large value of Control Gain. The receptance curves for a one degree of freedom coupled to the friction damper are used to evaluate the control strategies performances. All results were obtained by using the time domain integration procedure, called discrete-time statespace formulation, proposed by Lyan-Ywan [12]. Simulations were conducted by using physical parameters resulting from the identification process of the experimental test rig. The proposed modified control law, case " $\mathrm{D}$ ", is applied in this test rig and the results are compared with the numerical ones.

\section{2 theoretical approach}

The energy dissipation characteristics of a damper can be understood by its hysteresis loop. The hysteresis loop of a variable friction damper depends greatly on the control algorithm applied. In other words, the same variable friction damper with the same excitation may have different hysteretic behaviours when different control algorithms are applied. In this work, all hysteresis curves had been obtained using the friction damper model as presented by Fig. 1 .

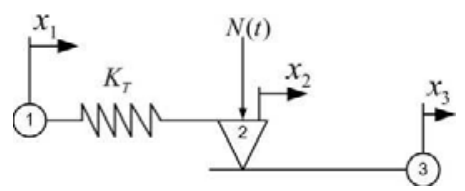

Fig. 1. Friction damper model.

For the proposed model the force between NODE 1 and NODE 3 is written as follow:

$F_{13}=\left\{\begin{array}{lr}K_{T}\left(x_{3}-x_{1}\right) & \text { if } K_{T}\left(x_{3}-x_{1}\right)<\mu N \\ \operatorname{sign}\left(x_{3}-x_{1}\right) \mu N & \text { if } K_{T}\left(x_{3}-x_{1}\right) \geq \mu N\end{array}\right.$
Assuming harmonic motion for $x_{1}(t)$, and $x_{3}(t)=$ $0 \forall t$ on equation (1), the normalized hysteresis cycles for the three studied cases were idealized and presented in the Fig. 2.

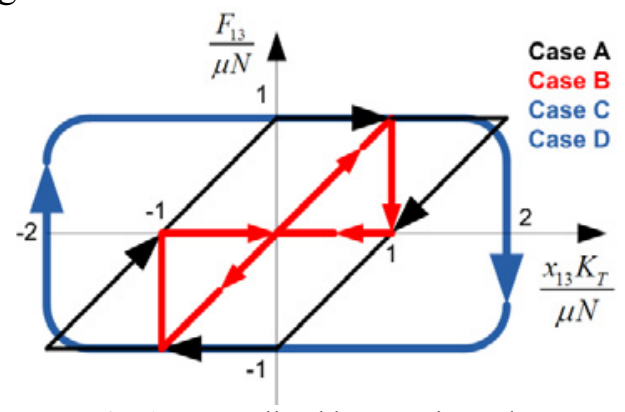

Fig. 2. Normalized hysteresis cycles.

A 1 DOF system is presented in Fig. 3 with mass $m_{1}=$ $4.274 \mathrm{~kg}, \quad$ stiffness $k_{1}=60.5 \mathrm{kN} / \mathrm{m}$, with $c_{1}=$ $33.175 \mathrm{Ns} / \mathrm{m}$ viscous damper. These values correspond to a damping factor of $\xi=3.26 \%$ and a natural frequency $f_{n}=18.94 \mathrm{~Hz}$. Tangential stiffness for the friction damper has been settled at $K_{T}=636.3 \mathrm{kN} / \mathrm{m}$ and friction coefficient was adjusted as $\mu=0.325$ in order to calculate and simulate all studied cases. These values represent the parameters identified from the test rig.

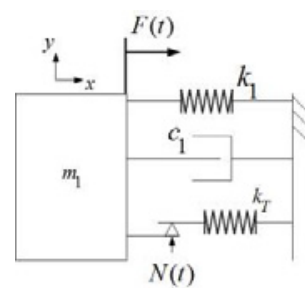

Fig. 3. One degree of freedom vibratory system.

These physical parameters, for the linear system, had been identified using the receptance curve measured on the test rig. It is possible to notice a good agreement between the experimental curve and the estimated one (Fig.4).

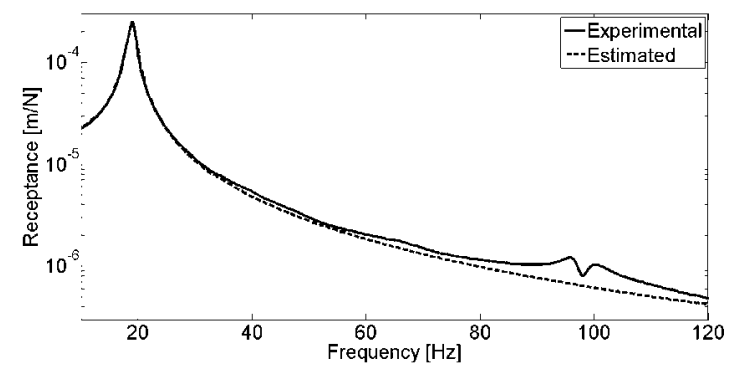

Fig. 4. Measured and estimated receptance curve.

Should be noticed the presence of small peaks between 90 and $100 \mathrm{~Hz}$. These peaks are due the test rig setup, which consist of a single base where is attached the 1 DOF vibratory system and the column to support the friction damper.

To identify the contact parameters, harmonic excitation of $20 \mathrm{~N} \mathrm{at} 19 \mathrm{~Hz}$ was applied to the system,. The 
frequency was chosen as close as possible to the resonant frequency. These conditions guarantee also the permanent slippage state to the friction damping even for small values of the normal force such as $10 \mathrm{~N}$. the hysteresis cycle was measured experimentally. This hysteresis cycle was used also to estimate the friction coefficient and the tangential stiffness by means of a curve fitting procedure of Eq. (1). In Fig. 5 is possible to see that the measurements present some noises especially for negative values of the friction force, ones can observe some differences between two curves close to stick regions due the stick/slip transition in this region which disturbs the measurements.

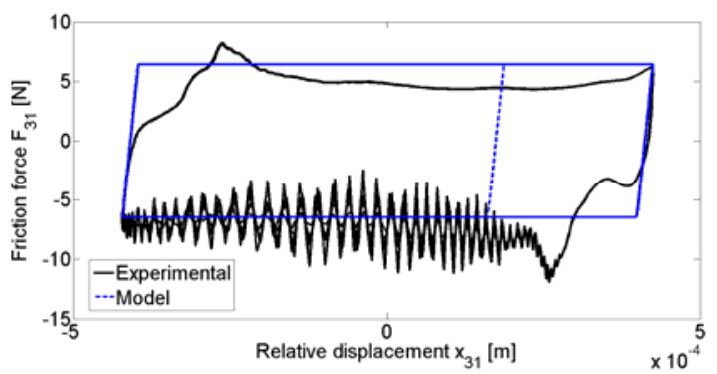

Fig. 5. Hysteresis Cycles.

\section{3 numerical results}

The control law strategies was studied as proposed by Santos \& Neto [13] and the equations for numerical and experimental implementation are:

CASE A

$N_{1}(t)=G|P[\Delta(t)]|$

\section{CASE B}

$N_{2}(t)=\left\{\begin{array}{l}G \mid P[\Delta(t)] \text { if } \operatorname{sign}\left(\frac{d \Delta(\mathrm{t})}{\mathrm{dt}} * \Delta(t)\right) \geq 0 \\ 0 \quad \text { if } \operatorname{sign}\left(\frac{d \Delta(\mathrm{t})}{\mathrm{dt}} * \Delta(t)\right)<0\end{array}\right.$

CASE C

$N_{3}(t)=\beta(G|P[\Delta(t)]|)\left|\tanh \left[\alpha \frac{d \Delta(t)}{d t}\right]\right|$

\section{CASE D}

$N_{4}(t)=\beta(G|P[\Delta(t)]|)|\tanh [\alpha V(t)]|$

For "Case D" Eq. (5) calculates the normal force as the modification of Eq. (4) as originally proposed by Santos and Neto [13]. The parameters $G, \Delta(t), \alpha$ and $\beta$ are respectively the control gain, the damper deformation $\left(x_{3}-x_{1}\right)$, the boundary layer parameter and an additional gain. Should be noticed that $\alpha$ and $\beta$ had been defined by Yang et al [9]. The operator $P$ [] gives the last variable $\Delta(t)$ peak or valley. The predictor $V(t)$ estimates the value of $d \Delta t / d t$ as:

$V(t)=\frac{d \Delta(t)}{d t}+\frac{d^{2} \Delta(t)}{d t^{2}} * d t$

To evaluate the performance of the proposed control laws, they were applied for the control of the 1 DOF vibratory system aforementioned excited with a $20 \mathrm{~N}$ harmonic force. The excitation frequency sweep has changed in steps of $0.25 \mathrm{~Hz}$ from $10 \mathrm{~Hz}$ up to $120 \mathrm{~Hz}$ to calculate the receptances. These results are shown in Fig. 6.

We can observe that the best performance has been achieved with the control law for "Case D". All control laws has been able to produce a high reduction in the receptance amplitude eliminating the resonance peak. However, Santos and Neto [13] comment that the lowest friction force is obtained with "Case D" control law which can represent a lowest level of contact surfaces wear.

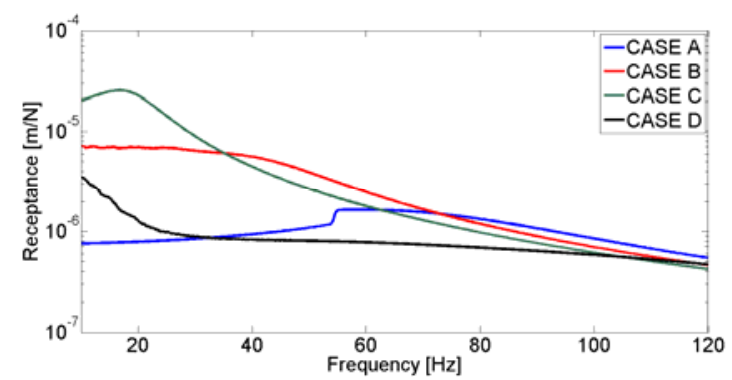

Fig. 6. Receptances obtained numerically for the 1 DOF controlled system.

\section{4 experimental results}

The test rig used to validate the numerical results and to study the friction damper is presented in the Fig. 7. In this figure is not presented the computer and the DAQ BOARD used to control the normal force. To control the excitation level, an Agilent Signal Analyser 35670A was used.

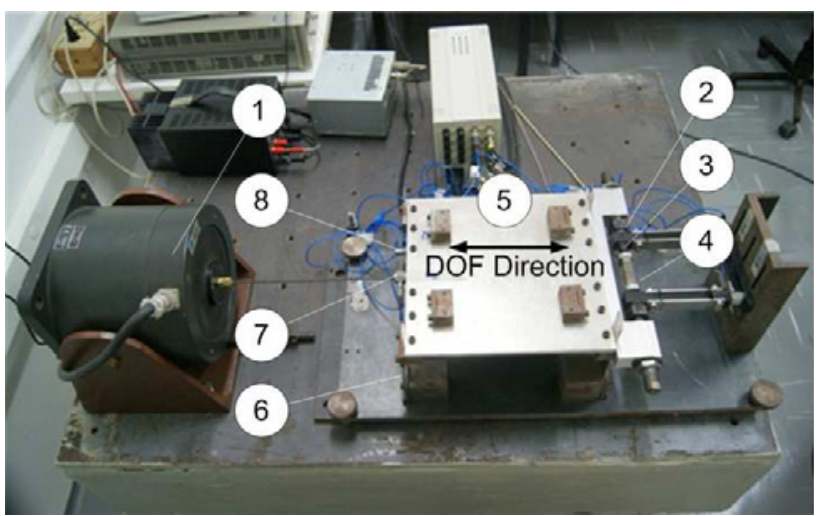

Fig. 7. Test rig setup. (1- Exciter, 2-Contact surface, 3-Normal force load cell, 4- Piezoelectric actuator, 5- DOF Mass, 6- DOF suspension, 7- Excitation load cell and 8-DOF accelerometer)

Using this setup, tests with constant normal forces were conducted in order to validate numerical simulations. The results obtained are shown in Fig. 8. 


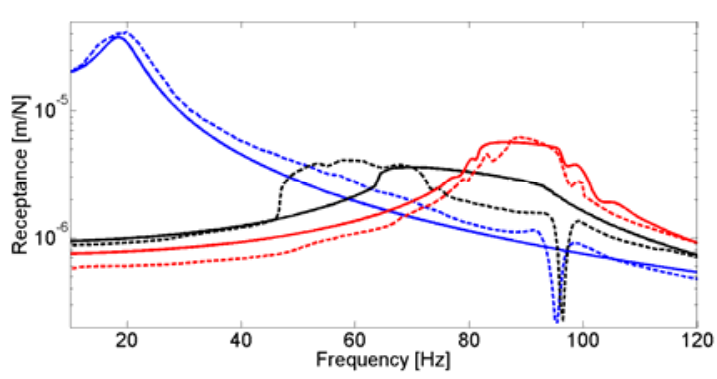

Fig. 8. Receptance obtained with constant normal forces. Dashed lines are experimental results and Solid ones numerical results. $\boldsymbol{F}_{\boldsymbol{e}} / \boldsymbol{F}_{\boldsymbol{N}}$ is equal to 0.1 for red lines, 0.5 for black lines and 2.0 for blue lines.

Should be noticed the good agreement between numerical and experimental results. The greater difference arises for the black lines. This difference can be due to the errors coming from noise in the instrumentations and also due to the difficulties to measure the displacement at low levels of response. Other important factor that contributes for the small deviations between both curves is the antiresonance closest to $95 \mathrm{~Hz}$, it can be originated in secondary structures of the test rig that are being stiffened. Besides, the numerical model is representative of the test rig dynamical behaviour. Regarding the relation $F_{e} / F_{N}$ the test rig setup has a limitation imposed by the exciter maximum force and the resolution of the control chain for $F_{e}$, i.e., for larger values of $F_{e} / F_{N}$ it is necessary larger values for $F_{e}$ which is limited by the exciter. On the other side, low levels of $F_{e}$ is truncated by the instruments resolution.

\section{Conclusions}

Increase the normal force $F N(t)$ for a passive damper reduces the resonance peak until a limit, after this limit the impedance will have a new resonance peak due to the parallel association of both stiffness: Tangential Stiffness and Suspension Stiffness.

Control law for Case $\mathrm{C}$ reduces the resonance peak almost 100 times. Although the resonance peak reduction, this control law has a poor performance. Case B and Case $\mathrm{C}$ had a similar performance. Case D control law can be considered as the best control law.

The controller $N_{4}(t)$ performance before the resonance peak should be improved giving for the system the good performance as in frequencies higher than the first resonance.

Future works will be conducted to optimize the friction damper behavior.

\section{References}

1. Arrao, A.S. and J.G. Nourse, Vibration Damping in Rotor Blades, in UK Patent Application 1990, General Electric Company: UK. p. 17.

2. Lu, L.-Y., G.-L. Lin, and T.-C. Kuo, Stiffness controllable isolation system for near-fault seismic isolation. Engineering Structures, 2008. 30(3): p. 747-765.

3. Dupont, P., P. Kasturi, and A. Stokes, SEMI-ACTIVE CONTROL OF FRICTION DAMPERS. Journal of Sound and Vibration, 1997. 202(2): p. 203-218.

4. Alvarez-Sánchez, E., A Quarter-Car Suspension System: Car Body Mass Estimator and Sliding Mode Control. Procedia Technology, 2013. 7(0): p. 208214.

5. Inaudi, J.A., MODULATED HOMOGENEOUS FRICTION: A SEMI-ACTIVE DAMPING STRATEGY. Earthquake Engineering \& Structural Dynamics, 1997. 26(3): p. 361-376.

6. Menq, C.H., J.H. Griffin, and J. Bielak, The influence of microslip on vibratory response, Part II: $A$ comparison with experimental results. Journal of Sound and Vibration, 1986. 107(2): p. 295-307.

7. Menq, C.H., J. Bielak, and J.H. Griffin, The influence of microslip on vibratory response, part I: $A$ new microslip model. Journal of Sound and Vibration, 1986. 107(2): p. 279-293.

8. Ozbulut, O.E., M. Bitaraf, and S. Hurlebaus, Adaptive control of base-isolated structures against near-field earthquakes using variable friction dampers. Engineering Structures, 2011. 33(12): p. 3143-3154.

9. He, W., A. Agrawal, and J. Yang, Novel Semiactive Friction Controller for Linear Structures against Earthquakes. Journal of Structural Engineering, 2003. 129(7): p. 941-950.

10. Nitzsche, F., et al., Development of a Maximum Energy Extraction Control for the Smart Spring. Journal of Intelligent Material Systems and Structures, 2005. 16(11-12): p. 1057-1066.

11. Vanderborght, B., et al., Variable impedance actuators: A review. Robotics and Autonomous Systems, 2013. 61(12): p. 1601-1614.

12. Lu, L.-Y., et al., Dynamic analysis of structures with friction devices using discrete-time state-space formulation. Computers \&amp; Structures, 2006. 84(15-16): p. 1049-1071.

13. Santos, M.B.d. and F.P. Lepore, Assessment on Control Strategies of Friction Dampers, in Word Tribology Congress 20132013, Associazione Italiana di Tribologia: Turim, Italy. 